\title{
Sistema de informação de transporte público coletivo no Brasil: algumas considerações sobre demanda de informação dos usuários em pontos de parada de ônibus
}

\author{
Information system for public transportation in Brazil: some considerations on users' \\ information demand at bus stops
}

Cristine O. Lanzoni; Cristiele A. Scariot; Carla G. Spinillo.

\begin{abstract}
transporte público, sistema de informação, ponto de parada
O presente artigo traz algumas considerações sobre a demanda por informação de usuários do serviço de transporte público coletivo nos pontos de parada de ônibus. Pela nova legislação brasileira de mobilidade urbana, o poder público deve prover a população, nos pontos de parada de embarque e desembarque, com informações sobre: itinerários; horários; tarifas dos serviços; e formas de interação com outros modais. Questiona-se neste artigo, então, se essas informações são suficientes para que o usuário possa realizar a tarefa de uso do transporte público coletivo de maneira eficaz. Os aspectos discutidos podem auxiliar o planejamento e design de sistemas de informação destinados a usuários do transporte público coletivo brasileiro.
\end{abstract}

public transportation, information system, bus stop

This article makes considerations on the information needs of users of public transportation service at bus stops. Under the new Brazilian legislation of urban mobility, the government should provide users at bus stops with information on itineraries, schedules, service prices and interaction with other transportation modes. So, this paper questions how sufficient is the information provided at bus stops to allow users to effectively accomplish the task of using public transportation in Brazil. The aspects discussed might support the planning and design of passenger information systems for Brazilian public transportation.

\section{Introdução}

O transporte público coletivo é um dos serviços considerados de caráter essencial à população (BRASIL, 1988). Apesar disso, numa visão geral, o transporte público coletivo no Brasil tem inovado pouco em relação à tecnologia, gestão e prestação de serviços aos usuários (DUARTE; LIBARDI; DUDUS, 2008). A opinião predominante dos brasileiros (31,3\%), quando perguntados sobre a qualidade do transporte público coletivo em suas cidades, é de que o serviço é "Regular" (INSTITUTO DE PESQUISA ECONÔMICA APLICADA, 2011).

Recentemente, o marco regulatório do transporte público coletivo no Brasil foi modernizado com a sanção da Lei no 12.587/2012, que institui diretrizes da Política Nacional de Mobilidade Urbana. Um dos pontos da nova lei de interesse para esta pesquisa é a obrigação do poder competente em informar a população acerca do serviço de transporte público coletivo. Assim, passa a ser direito do usuário ser informado nos pontos de embarque e desembarque de passageiros, de forma gratuita e acessível, sobre: itinerários; horários; tarifas dos serviços; e modos de interação com outros modais (BRASIL, 2012).

Ainda, pela nova lei, o usuário do transporte público coletivo tem o direito de ser informado, em linguagem acessível e de fácil compreensão, sobre: seus direitos e responsabilidades; direitos e obrigações dos operadores dos serviços; padrões pré-estabelecidos de qualidade e quantidade dos serviços ofertados; e meios para reclamações e seus respectivos prazos de resposta (BRASIL, 2012). 
Apesar da louvável preocupação da nova lei com a presença e a linguagem de certas informações aos usuários do transporte público coletivo, questiona-se neste artigo se as informações tidas como obrigatórias nos pontos de parada são suficientes para suprir a demanda de informação dos usuários na utilização do serviço.

Assim, o artigo traz um estudo sobre as possíveis demandas de informação que um usuário do serviço de transporte público coletivo possui nos pontos de parada. Para atingir tal objetivo, o estudo parte de um levantamento bibliográfico para compreender o contexto do serviço de transporte público coletivo no Brasil, o processo de orientação espacial dos indivíduos e a relação dessas temáticas com sistemas de informação. E em seguida, faz uso da ferramenta de decomposição da tarefa para compreender a tarefa e as demandas de informação que um usuário possui durante seu processo de orientação e deslocamento pela cidade utilizando o serviço de transporte público coletivo por ônibus.

\section{Serviço de transporte público coletivo}

O Brasil consolida-se como um país notavelmente urbano, com $84,4 \%$ de sua população vivendo em áreas urbanas (IBGE, 2010). A busca pelo espaço urbano e, portanto, seu crescimento, demanda da gestão pública constante planejamento, instituição de políticas urbanas, criação de infra-estrutura e oferta de serviços públicos, ante as necessidades e os interesses cotidianos dos cidadãos, visando o bem-estar de todos (BRASIL, 2001).

No que tange a oferta de serviços públicos, o transporte público coletivo é tido como um serviço essencial, definido como o transporte de passageiros acessível a toda população mediante pagamento individualizado, com itinerários e preços fixados pelo poder público (BRASIL, 1988, 2012).

Segundo a Constituição Federal, compete aos Municípios organizar e prestar, diretamente ou sob regime de concessão ou permissão, o serviço de transporte público coletivo. A recente Lei $n^{\circ}$ 12.587/2012, que institui diretrizes da Política Nacional de Mobilidade Urbana, regulamenta novos princípios, diretrizes e instrumentos a serem executados pelos Municípios na defesa dos interesses dos usuários dos serviços de transporte público coletivo (BRASIL, 1988, 2012).

Segundo pesquisa, o transporte público coletivo é o meio de transporte mais utilizado pelos brasileiros dentro da cidade $(44,3 \%)$, seguido pelo carro $(23,8 \%)$, por moto $(12,6 \%)$, a pé $(12,3 \%)$ e, por último, pela bicicleta $(7,0 \%)$ (INSTITUTO DE PESQUISA ECONÔMICA APLICADA, 2011).

Nas cidades brasileiras, a integração de transporte público coletivo mais utilizada é a de ônibus/ônibus (INSTITUTO DE PESQUISA ECONÔMICA APLICADA, 2011); sendo que algumas cidades contam, também, com modais como metrô, trem, e, mais recentemente, com vans e outros veículos de pequena capacidade. Independente dos tipos de veículos utilizados no serviço de transporte público coletivo espera-se que esse seja prestado de forma profissional, não admitindo riscos de descontinuidade e com uma adequada organização de seus processos de trabalho, para oferecer o máximo de produtividade, o mínimo de agressão ao meio ambiente e o melhor atendimento à população, com conforto, fluidez e segurança (SECRETARIA NACIONAL DE TRANSPORTE E DA MOBILIDADE URBANA, 2007).

Para a obtenção de um serviço de qualidade, é fundamental a conciliação de interesses de três grupos com preocupações distintas quanto ao desempenho de um sistema de transporte público, sendo eles (EMPRESA BRASILEIRA DE TRANSPORTES URBANOS, 1988):

- Usuários: utilizam o serviço público para suprir suas necessidades de deslocamento e não tem maiores preocupações com a operação dos serviços. Na tomada de decisão quanto ao uso do transporte público, levam em consideração a regularidade, tempo de deslocamento, conforto, custos, entre outros atributos.

- Operadores: encarregados de administrar, operar (financiamento, aquisição, manutenção, renovação da frota, etc.) e comercializar o transporte coletivo, sob a forma de prestação de um serviço público. Suas preocupações estão relacionadas com as 
variáveis que influenciam os custos e receitas na oferta do serviço.

- Poder público: responsável legal pelo transporte público; deve regulamentar, planejar, programar e fiscalizar a execução dos serviços, interagindo nos conflitos de interesse entre usuários e operadores através de legislação específica. Fica, também, a cargo do poder público defender os interesses de um quarto grupo, a comunidade em geral, cujo interesse é indireto, provocado pelas externalidades do sistema de transporte publico (ruído excessivo, poluição ambiental, conflitos com o uso do solo, etc.).

\section{O processo de orientação espacial e sistemas de informação}

Estar orientado, segundo Bins Ely (2003), significa saber onde se está, no espaço e no tempo, e poder definir seu próprio deslocamento. O processo de orientação espacial, para a mesma autora, está intimamente relacionado com as características de cada indivíduo, suas experiências e a capacidade do espaço em oferecer informação através de elementos e sistemas de informação.

Os elementos e sistemas de informação ambientais, conforme Bins Ely (2003), variam em função da escala do ambiente em questão e devem estar relacionados entre si para uma maior eficiência, sendo eles:

- Informação arquitetônica: refere-se à configuração espacial, à presença ou não de elementos referenciais e ao zoneamento funcional;

- Informação do objeto: refere-se às características particulares do objeto e suas relações com o ambiente, responsável pela identificação do objeto e sua função de uso. O reconhecimento desse tipo de informação está relacionado a fatores culturais e conhecimento prévio do objeto/função por parte do individuo;

- Informação adicional: complementa às duas outras modalidades, pode ser transmitida através de suportes físicos permanentes ou transitórios locados em um espaço (placas, sinais, displays, mapas, banners); fornecida para uso individual (folhetos, brochuras, etc.); transmitida de forma sonora (apitos, sirenes); verbal (informações por funcionários ou usuários) ou tátil (sinalização no piso através de textura).

Para Arthur e Passini (2002), o processo de orientação espacial abrange três aspectos: 1) a tomada de decisão, referente ao planejamento das ações; 2 ) a execução da decisão, que é a transformação do plano em ações, e 3) o processamento da informação, que corresponde à percepção do ambiente e a transformação das informações em imagem ambiental. Esses três aspectos se inter-relacionam para dar condições ao indivíduo de se movimentar de forma orientada.

Para Kishnani (1994), o processo de orientação espacial envolve primeiramente, o conhecimento pelo usuário da tarefa a ser executada e o local de destino. Em seguida, o ambiente deve fornecer ao indivíduo informações para que este possa reconhecer o local onde se encontra - ponto de origem - e o local onde deseja chegar - ponto final. Com essas informações o usuário pode definir, escolher e executar seu trajeto.

Conforme Satalich (1995), o processo de orientação espacial de um usuário pode ser descrito em quatro passos, conforme a figura 1 a seguir: 
FIGURA 1. Modelo de processo de orientação espacial. Fonte: Scariot; Lanzoni; Spinillo (2011) baseado em Satalich (1995).

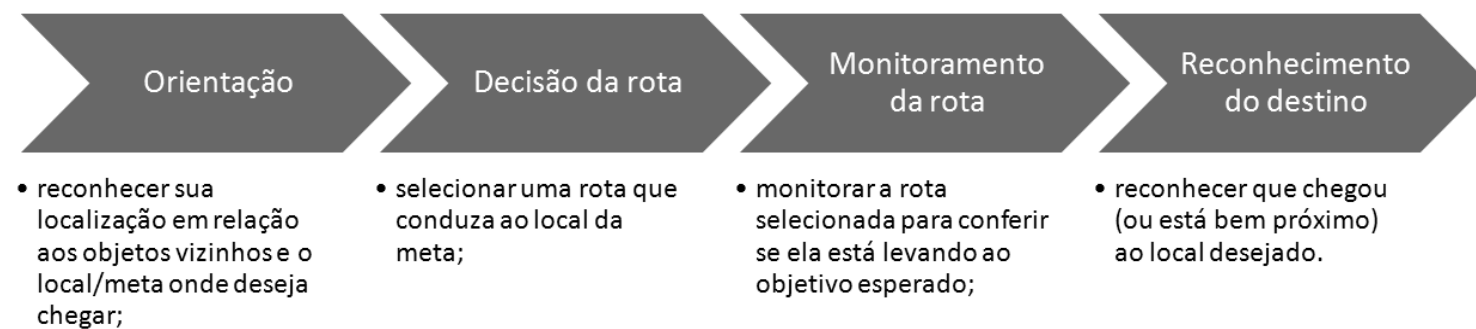

Compreender as etapas do processo de orientação espacial, levantadas por diferentes autores e citadas acima, mostra-se bastante pertinente ao design de um sistema de informação para usuários de transporte público coletivo, uma vez que a presença de um sistema de informação pode auxiliar diretamente o usuário na execução dessas etapas.

\section{Sistema de informação para usuários de transporte público coletivo}

O objetivo de um sistema de informação para usuários do transporte público, para Cutolo (2003), é a promoção da mobilidade, pois a informação permite que as pessoas planejem e definam seus deslocamentos pela cidade.

$\mathrm{Na}$ concepção de um sistema de informação, os responsáveis pelo transporte público coletivo tendem a cometer dois erros: o primeiro é o excesso de informações, que não tornam, necessariamente, o sistema útil e fácil de usar; o segundo erro, em contrapartida, é a escassez de informação, em decorrência da suposição errônea que os usuários comuns já conhecem o sistema ou o entendem intimamente (EMBARQ, 2002).

Segundo Silva (2000), os sistemas de informações aos usuários garantem um aumento de qualidade do serviço ofertado aos passageiros, e devem visar a: atualização e confiabilidade das informações; compreensão das informações pelos usuários; amplitude de cobertura ou o alcance das informações; e rapidez/objetividade no provimento das informações.

Ainda Silva (2000) define que um sistema de informação ao usuário pode ser usado no transporte público para exercer diferentes funções, sendo elas:

- Promocional: relacionada à promoção de motivos e destinos de viagens e à informação de disponibilidade dos serviços, visa melhorar a imagem do transporte público e promover sua utilização entre a população;

- De instrução: volta-se à função de divulgar as regras de utilização transporte público e orientar seu melhor uso;

- Operacional: permite que o usuário planeje sua viagem, tenha acesso ao sistema de transporte, realize sua viagem e saiba de possíveis restrições ou alterações na programação; e

- De moderação: atua no comportamento do passageiro, diminuindo sua ansiedade e dando a ele maior controle de escolha entre as opções disponíveis.

Segundo Molinero e Sanchez (1998), um sistema de informação ao usuário precisa atender diferentes grupos de usuários, que apresentam necessidades de informação variadas, de acordo com sua relação com o sistema de transporte público coletivo; sendo eles:

- Usuários regulares em rota cotidiana: devido sua maior familiaridade com o serviço, demandam menos informação sobre o uso do serviço, mas continuam suscetíveis ao recebimento de comunicados acerca de alterações no funcionamento do sistema e das linhas de seu interesse; 
- Usuários regulares em rota nova: demandam informação sobre a rede de transporte e seus itinerários;

- Usuários potenciais: necessitam de informações acerca das tarifas, horários e rotas do sistema de transporte coletivo. Ainda, as informações promocionais podem ter grande influência sobre esses usuários, motivando-os a utilizar o serviço; e

- Usuários turistas: desconhecem as condições locais, portanto, necessitam de informações mais completas e detalhadas sobre o serviço prestado e sobre a cidade.

O conceito de sistema de informação implica a presença de um conjunto de componentes que se inter-relacionem de forma a oferecer ao usuário o máximo de informação sobre o transporte público. Tais componentes podem ser classificados quanto: ao tipo de informação desejada; a localização da informação; e a forma de distribuição desta (FERNANDES, 2007; VIEIRA, 2000; FERRAZ E TORRES, 2001).

Com relação ao tipo de informação, Fernandes (2007) analisa como sendo oito os tipos de informação desejada, detalhadas na figura 2: informações gerais sobre a rede; identificação do serviço; serviços especiais e ocasionais; itinerários; horários; tarifas; informações diversas; e regras de operação.

FIGURA 2. Tipos de informação desejada. Fonte: Scariot; Lanzoni; Spinillo (2011) baseado em Fernandes (2007).

\section{Informações \\ gerais sobre a rede}

\section{Identificação \\ do serviço}

\section{Serviços
especiais e \\ Serviços
especiais e \\ ocasionais}

Itinerários

Itinerários

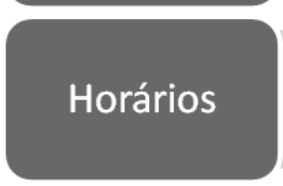

- informações a respeito dos trajetos das linhas, podendo ser representados por ruas, mapas, esquemas.
- características gerais de médio e longo prazo, a respeito dos serviços ofertados, venda de passagens, tarifas, condições para uso, serviços da rede normal, serviços aos domingos, freqüências, tempos de percurso.

- informações que devem ter sua divulgação prevista pela rede, tais como: nome da parada; nome e número da linha; identificação do operador; direção/destino da linha; itinerário; e paradas.
- horário de chegado do ônibus na parada; horário de funcionamento da linha; horários de toda a rede; horários referentes a serviços ocasionais.

Tarifas

- preço das passagens, zonas tarifárias, descontos, multas e isenções.

\section{Informações}

diversas

Regras de operação
- Complementam outras informações: localização de pontos turísticos; números de emergência; contato para sugestões e reclamações. 
Em termos de localização da informação, segundo Vieira (2000), as informações podem ser ofertadas nos seguintes locais: moradia do usuário; espaços públicos; terminais; pontos de parada; e veículos. Para Ferraz e Torres (2001), as informações podem ser distribuídas em diferentes áreas de uma mesma localidade, através de diferentes dispositivos (e.g. folhetos, painéis digitais, caixa de itinerários), como visto na figura 3.

FIGURA 3. Localização e distribuição da informação. Fonte: Scariot; Lanzoni; Spinillo (2011) baseado em Ferraz e Torres (2001).
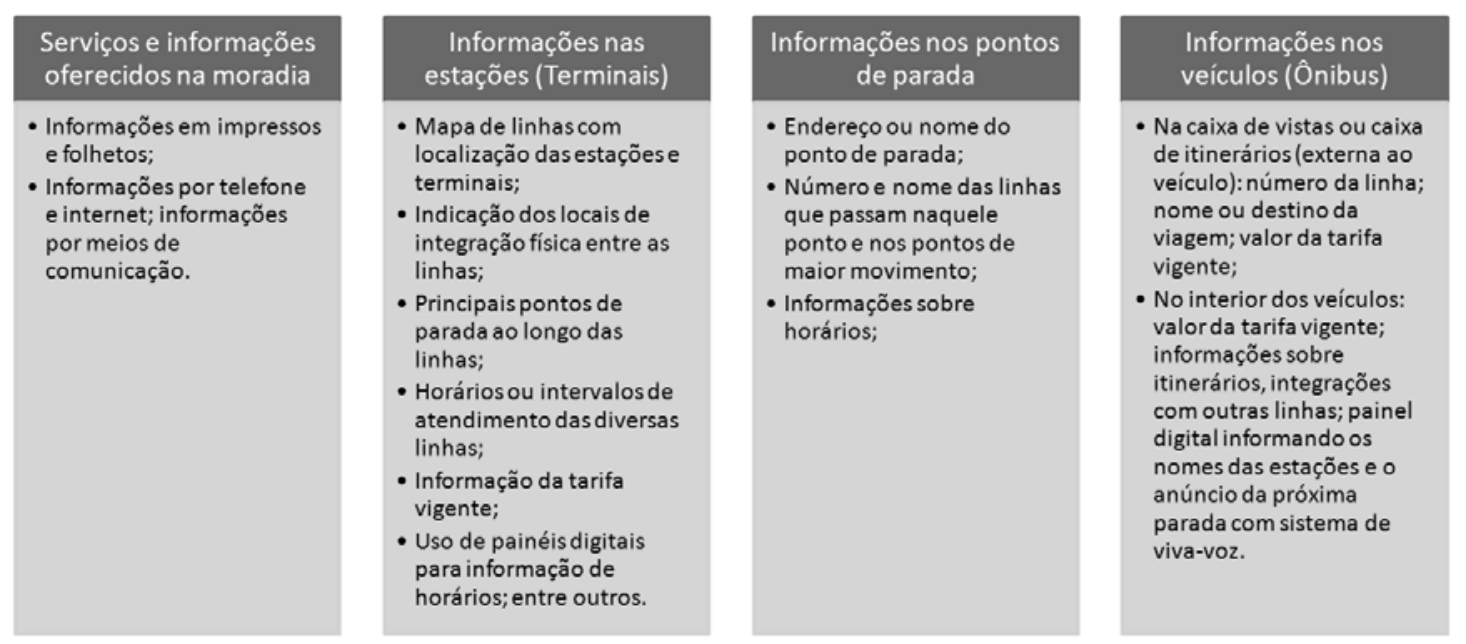

Além da presença de informações pertinentes em locais adequados, outro aspecto relevante no design de um sistema de informação ao usuário do transporte público coletivo é a adequação das informações e da apresentação gráfica dessas informações ao contexto social, econômico, local, e de familiaridade do leitor com a linguagem verbal e visual da mensagem estando este último diretamente relacionado a certas características como idade, cultura e escolaridade (WRIGHT, 1999; SPINILLO, 2002).

Segundo Wright (2003), a efetivação da informação ocorre quando o usuário consegue, a partir de uma rápida observação do material, encontrar, compreender e aplicar as informações disponibilizadas.

\section{Identificando demanda de informação dos usuários em pontos de parada}

Entre as localidades em que a informação pode ser ofertada ao usuário, vistas anteriormente, encontram-se os pontos de parada, definidos como locais nas vias públicas onde se realiza a parada de veículos de transporte coletivo para o embarque e/ou desembarque de passageiros (ASSOCIAÇÃO NACIONAL DE TRANSPORTES PÚBLICOS, 1995). Segundo pesquisa do Instituto de Pesquisa Econômica Aplicada (2011), o ponto de parada, com média nacional de $31 \%$, é a principal fonte de informação das pessoas sobre transportes nas cidades.

A fim de reconhecer as possíveis demandas de informação dos usuários do transporte público coletivo nos pontos de parada, foi realizada pelas autoras a decomposição seqüencial da tarefa cognitiva de utilização do transporte público coletivo, de acordo com o proposto por Moraes e Mont'Alvão (1998). Isto permitiu conhecer a estrutura da tarefa, suas atividades e a seqüência em que essas ocorrem.

Na decomposição da tarefa do tipo seqüencial, esta é desmembrada em atividades bastante específicas, representadas em um fluxograma que segue exatamente a ordem em que ocorrem. As atividades da tarefa que demandam uma ação são representadas graficamente por retângulos e aquelas relacionadas a uma tomada de decisão por um losango. Estas últimas encadeiam novas ações excludentes entre si, dependendo da resposta do usuário, sendo: (S) Sim e (N) Não. 
O fluxograma resultante da decomposição seqüencial da tarefa cognitiva de utilização do transporte público coletivo por usuários encontra-se na figura 4 a seguir. Com isto identificou-se que a tarefa envolve, ao todo, sete tomadas de decisão e cinco possíveis ações, complementares ou excludentes entre si (e/ou) por parte do usuário. Nos momentos de tomada de decisão (e.g. o usuário identifica o ponto de parada?), caso o usuário tenha conhecimento prévio das informações necessárias para a realização da tarefa, poderá realizá-la com autonomia até sua finalização. Entretanto, a falta de conhecimento necessário para a tomada de decisões, levará o usuário a agir na busca de satisfazer as necessidades informacionais (e.g. consultar outras pessoas; desistir de utilizar o serviço). Uma das ações possíveis do usuário é a consulta a sistemas de informação (e.g., placas, totens informativos), caso existam.

Cada tomada de decisão do usuário na tarefa pode ser auxiliada por informações, sejam elas arquitetônicas, do objeto ou adicionais. Sendo assim, foram indicados no fluxograma de decomposição da tarefa do tipo seqüencial, exemplos de informações que podem auxiliar o usuário nos diferentes momentos da tarefa. A seqüência das atividades da tarefa acaba por seguir as etapas do processo de orientação, descrito anteriormente, de tal modo que as atividades podem ser locadas em três momentos distintos, também apontados na figura 4:

- No ponto de parada (de embarque): onde ocorrem as quatro primeiras tomadas de decisão pelo usuário, relacionadas ao reconhecimento de sua localização e destino, e da definição de sua rota;

- Dentro do veículo (ônibus): onde ocorrem duas tomadas de decisão pelo usuário relacionadas ao monitoramento de sua rota;

- No ponto de parada (de desembarque): onde ocorre a última tomada de decisão pelo usuário, relacionada ao reconhecimento de sua localização.

FIGURA 4. Decomposição seqüencial da tarefa cognitiva de utilização do transporte público coletivo por usuários. Fonte: Scariot; Lanzoni; Spinillo (2011)

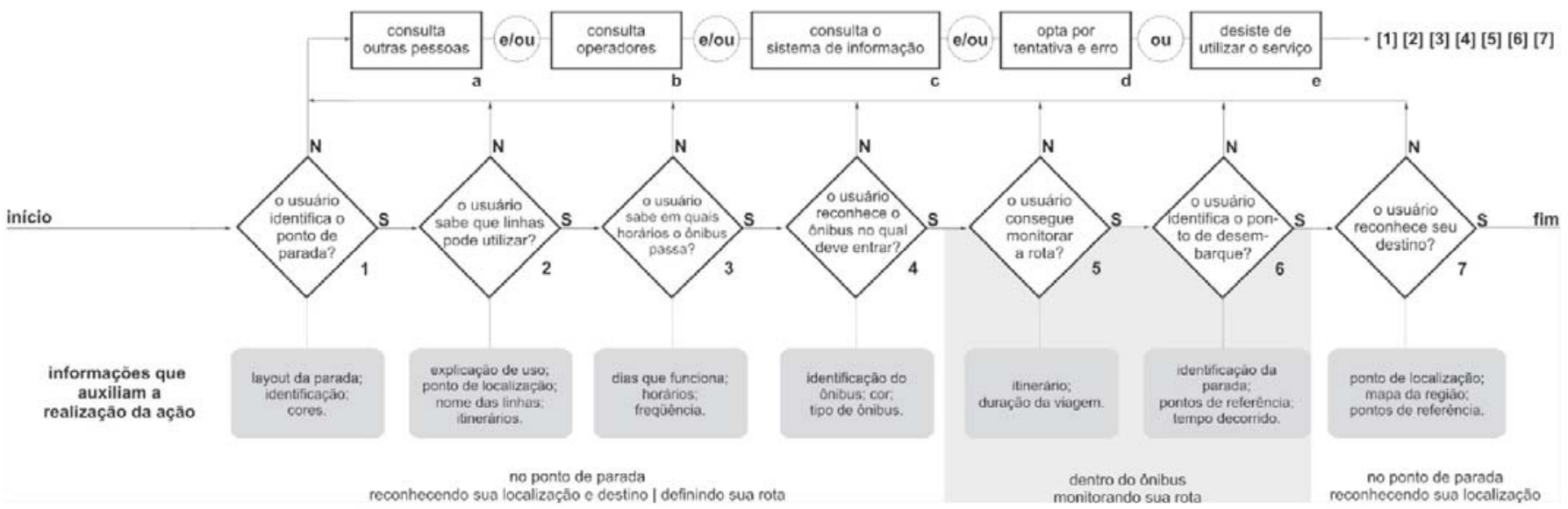

Os aspectos identificados a partir da decomposição da tarefa, em conjunto com os demais dados previamente apontados neste artigo, permitiram apontar algumas demandas de informação operacional de usuários do transporte público coletivo, dos usuários regular ao turista. Estas referem-se tanto às paradas de ônibus como aos veículos para a utilização do serviço de transporte público, sendo:

1. Identificar o mobiliário urbano como sendo um ponto de parada;

2. Identificar o serviço de transporte público ofertado naquele ponto de parada e o tipo de veículo utilizado;

3. Reconhecer as linhas que param naquele ponto de parada;

4. Visualizar o itinerário completo de todas as linhas disponíveis naquela parada; 
5. Visualizar todos os pontos de parada existentes no itinerário das linhas;

6. Reconhecer nos itinerários disponíveis o destino final de sua viagem e seu ponto de parada de desembarque;

7. Identificar outras linhas disponíveis nas proximidades, caso não encontre uma linha favorável a si naquela parada;

8. Identificar o tempo de espera para a chegada do veículo;

9. Relembrar e reavaliar o itinerário, após o embarque no veículo;

10. Monitorar o percurso realizado pelo veículo, para identificar se seu ponto de desembargue está próximo;

11. Planejar, durante a viagem de ida, sua viagem de volta com a mesma linha.

12. Reconhecer sua localização geográfica na cidade, ao desembarcar do veículo, tendo como ponto de partida/referência a parada de ônibus, para deslocar-se até seu destino final;

13. Identificar outras fontes de informação disponíveis, caso não encontre as informações necessárias a si naquela parada ou veículo.

Esses aspectos podem vir a ser utilizados para embasar e direcionar o planejamento e desenvolvimento de sistemas de informação para usuários do transporte público coletivo. Assim, podem contribuir para satisfazer as possíveis demandas de informação dos usuários durante o processo de orientação e deslocamento pela cidade utilizando o transporte público coletivo.

\section{Considerações finais}

O transporte público coletivo é um serviço essencial à população brasileira e precisa agregar qualidade aos seus serviços de forma a atender as necessidades dos usuários. Isto se dá especialmente diante do crescente uso do transporte individual privado, o qual pode ser considerado inviável tanto no sentido socioeconômico quanto ambiental para as cidades que pretendem desenvolver-se de forma justa e sustentável.

A presença de um sistema de informação destinado a usuários do transporte público coletivo pode melhorar tanto a qualidade quanto a imagem do serviço junto aos usuários e nãousuários, aumentando, assim, sua demanda e faturamento. $E$ ao informar e instruir, um sistema de informação possibilita que o usuário compreenda e utilize melhor o serviço, tornando-o realmente acessível a toda população.

Sendo assim, é necessário que o poder público, diretamente ou indiretamente, passe a considerar sistemas de informação para usuários como uma estratégia do transporte público coletivo. Esta sendo capaz de manter usuários atuais, bem como atrair novos; e invista no design de sistemas de informação que ajudem os usuários a tomar decisões mais eficientes.

A nova lei de mobilidade urbana (Lei no 12.587/2012) marca um importante avanço no reconhecimento pelo governo da importância da informação para os usuários do transporte público coletivo, ao instituir certas obrigatoriedades e recomendações quanto ao tipo de informação e linguagem a ser utilizada.

Com relação aos pontos de parada, passa a ser obrigatório informar nessas localidades, de forma gratuita e acessível, sobre: itinerários; horários; tarifas dos serviços; e modos de interação com outros modais. A partir do estudo realizado neste artigo, conclui-se que as informações previstas na nova legislação são imprescindíveis para a utilização do serviço pela população, mas que a demanda de informação dos usuários vai além dessas informações e mostra-se mais específica.

Os pontos de parada, portanto, devem ser utilizados para prover informações - tanto arquitetônicas, do objeto e adicionais - que permitam ao usuário, além da definição de sua rota, o reconhecimento de sua localização e destino. Além disso, para que o usuário possa 
completar seu processo de orientação e deslocamento utilizando o serviço de transporte público coletivo, é indispensável, também, a presença de informações no interior dos veículos (ônibus ou outros modais) - tornando possível ao usuário monitorar e alterar sua rota. Além dos pontos de parada e veículos, citados neste estudo, a oferta de informações para os usuários em suas moradias, nos espaços públicos e em terminais/estações também deveria ser prevista pelo serviço de transporte público coletivo.

Ressalta-se que o desenvolvimento de um sistema de informação ao usuário do transporte público coletivo é um projeto complexo que envolve diversas especificidades. Para evitar que o sistema de informação desenvolvido falhe pela falta de informações ou pelo excesso delas, recomenda-se, como demonstrado neste artigo, que os objetivos e necessidades de informação dos usuários sejam estudados a priori. Além disso, para garantir a qualidade e eficácia do sistema de informação, é de extrema importância que seu planejamento, desenvolvimento e operação sejam realizados de forma cuidadosa e profissional, de preferência com o envolvimento do usuário em todas as suas fases.

\section{Referências}

ARTHUR, P., PASSINI, R. Wayfinding: People, Signs, and Architecture. Toronto, New York: McGraw Hill, 1992.

ASSOCIAÇÃO NACIONAL DE TRANSPORTES PÚBLICOS. Pontos de parada de ônibus urbano: Caderno técnico №. 2. São Paulo: ANTP, 1995.

BINS ELY, V. Ergonomia + Arquitetura: buscando um melhor desempenho do ambiente físico. Anais do 3ํㅡㄹodesign. Rio de Janeiro: LEUI/PUC-Rio, 2003.

BRASIL. Constituição (1988). Constituição [da] Republica Federativa do Brasil. Brasília, DF: Senado Federal. Disponível em: <http://www.planalto.gov.br/ccivil_03/constituicao/ constitui\%C3\%A7ao.htm>. Acesso em: 20 jan. 2012.

BRASIL. Estatuto da Cidade. Lei $n^{\circ} 10.257$, de 10 de julho de 2001. Diário Oficial [da] República Federativa do Brasil, Brasília, DF, 11 jul. 2001. Disponível em: <http://www.planalto.gov.br/ccivil_03/leis/LEIS_2001/L10257.htm>. Acesso em: 20 jan. 2012.

BRASIL. Lei $n^{\circ}$ 12.587, de 03 de janeiro de 2012. Diário Oficial [da] República Federativa do Brasil, Brasília, DF, 03 jan. 2012. Disponível em: <http://www.planalto.gov.br/ ccivil_03/_ato2011-2014/2012/lei/l12587.htm>. Acesso em: 01 mar. 2012.

CUTOLO, F. Diretrizes para Sistemas de Informação ao Usuário. Palestra no III Seminário Internacional PROMOTEO. Porto Alegre, 2003.

DUARTE, F; LIBARDI, R; DUDUS, K. Introdução à mobilidade urbana. Curitiba: Juruá, 2007.

EMBARQ. De cá para lá: um guia criativo de marketing BRT para atrair e cativar usuários. EMBARQ-Brasil, 200?. Disponível em: < http://www.embarqbrasil.org/ >. Acesso em: 01 mar. 2012.

EMPRESA BRASILEIRA DE TRANSPORTES URBANOS. Planejamento e Operação: Elementos Intervenientes, v. 2. Ebtu: Brasília, DF, 1998.

FERNANDES, R.. Componentes gráficos para um sistema de informação visual em terminais de integração metrô-ônibus. Dissertação (Mestrado em Programa de Pós-graduação em Transportes) Universidade de Brasília, 2007.

FERRAZ, A.; TORRES, I. Transporte público urbano. Editora Rima. São Carlos, 2001.

INSTITUTO BRASILEIRO DE GEOGRAFIA E ESTATÍSTICA - IBGE. Sinopse do Censo Demográfico 2010. Rio de Janeiro, 2011. Disponível em: <http://www.ibge.gov.br/home/ estatistica/populacao/censo2010/sinopse.pdf >. Acesso em: 16 fev. 2012.

INSTITUTO DE PESQUISA ECONÔMICA APLICADA. Sistema de Indicadores de Percepção Social (SIPS): Mobilidade urbana. Brasília, 2011.Disponível em: <http://www.ipea.gov.br/ portal/images/stories/PDFs/SIPS/110124_sips_mobilidade.pdf>. Acesso em: 20 jan. 2012. 
KISHNANI, N. Space, signs, information. An evaluation of the wayfinding system at Stansted Airport. Proceedings of Public Graphics, p 22.1 - 22.16. The Netherlands: September 1994.

MOLINERO, A.; SANCHEZ, I. Transporte Público: Planeación, Diseño, Operación y Administración, 3a Edición. México: Fundación ICA,1998.

MORAES, A., MONTALVÃO, C. Ergonomia: conceitos e aplicações. Rio de Janeiro: 2AB, 1998.

SATALICH, G. A. Navigation and Wayfinding in Virtual Reality: Finding Proper Tools and Cues to Enhance Navigation Awareness. Master Thesis, University of Washington, 1995.

SCARIOT, C. A. ; LANZONI, C.; SPINILLO, C. G. O processo de orientação espacial e as necessidades informacionais de um usuário do transporte público. In: 5 CONGIC Congresso Nacional de Iniciação Científica em Design da Informação, 2011, Florianópolis. 5 Congresso Internacional de Design da Informação, 2011.

SECRETARIA NACIONAL DE TRANSPORTE E DA MOBILIDADE URBANA. PlanMob: construindo a cidade sustentável. Brasília: Ministério das Cidades, 2007. Disponível em: $<$ http://www.cidades.gov.br/images/stories/ArquivosSEMOB/Biblioteca/LivroPlanoMobilidade .pdf>. Acesso em: 20 jan. 2012.

SILVA, D. Sistemas Inteligentes no transporte público por ônibus.143 f. Dissertação (Mestrado em Engenharia de Produção) - Departamento de Engenharia de Produção, Universidade Federal do Rio Grande do Sul, Porto Alegre, 2000.

SPINILLO, C. G. Are visual instructions successful messages? Some considerations in the analysis of procedural pictorial sequences. In: GRIFFIN, R.; LEE, J.; WILLIAMS, V.(Org.). Visual literacy in message design: Selected Readings of the International Visual Literacy Association (IVLA) Annual Conference 2001. 1 ed. Loretto, PA: IVLA- International Visual Literacy Association, 2002, p. 01-10.

VIEIRA, L. F. Sistemas de informação ao usuário: Avaliando as preferências dos usuários da Bacia Operacional Sul - Porto Alegre. Anais do XIV congresso da ANPET, p. 215 - 226, 2000.

WRIGHT, P. Comprehension of printed instructions: examples from health materials. In: D. Wagner, R. Venezky \& B. Street (Eds.) Literacy: an international handbook. Boulder, CO: Westview Press, 1999, p. $192-198$.

WRIGHT, P. Criteria and ingredients for successful patient information. Journal of Audiovisual Media in Medicine, 26, 1, pp. 6-10. London: Taylor \& Francis. 1999, 2003.

\section{Sobre as autoras}

Cristine Lanzoni, Bacharela em Design Gráfico pela Universidade Federal do Paraná, é atualmente aluna do Mestrado em Design de Sistemas de Informação do PPGDesign UFPR. Tem como principais áreas de pesquisa o design da informação, o ambiente urbano e sistemas de transporte público.

cristinelanzoni@gmail.com

Cristiele Scariot, Bacharela em Design Gráfico pela UFPR, é atualmente mestranda do PPGDesign UFPR e tem como principais áreas de pesquisa o design da informação, sistemas complexos de informação visual e o design centrado no usuário.

cristielescariot@gmail.com

Carla Galvão Spinillo, Doutora em Typography Graphic Communication - University of Reading, Inglaterra, é bolsista de Produtividade em Pesquisa do CNPq [nível 2] com posdoutorado na University of Avans, Holanda. Atualmente é professora adjunto do PPGDesign da UFPR.

cgspin@gmail.com 